\title{
DNA methylation of metallothionein genes is associated with the clinical features of renal cell carcinoma
}

\author{
RUTA MALECKAITE ${ }^{1}$, ALGIRDAS ZALIMAS ${ }^{1,2}$, ARNAS BAKAVICIUS ${ }^{2}$, FELIKSAS JANKEVICIUS ${ }^{2}$, \\ SONATA JARMALAITE ${ }^{1,2}$ and KRISTINA DANIUNAITE ${ }^{1,2}$ \\ ${ }^{1}$ Institute of Biosciences, Life Sciences Center, Vilnius University, LT-10257 Vilnius; \\ ${ }^{2}$ National Cancer Institute, LT-08406 Vilnius, Lithuania
}

Received October 4, 2018; Accepted March 21, 2019

DOI: $10.3892 /$ or.2019.7109

\begin{abstract}
Metallothioneins are low-weight cysteine-rich proteins responsible for metal ion homeostasis in a cell and, thus, capable of regulating cell proliferation and differentiation. Deregulation of metallothionein genes has been reported in various human tumors. However, their role in renal cell carcinoma (RCC) has been poorly investigated. In the present study, we aimed to evaluate the importance of promoter DNA methylation of selected metallothionein genes for RCC. Based on the initial analysis of kidney renal clear cell carcinoma dataset from The Cancer Genome Atlas, genes $M T 1 E, M T 1 F, M T 1 G$ and $M T 1 M$ were selected for qualitative methylation analysis in 30 tumors (including 10 multifocal cases), 10 pericancerous, and 30 non-cancerous renal tissues (NRT). Methylation of MT1E and MT1M was tumor-specific $(\mathrm{P}=0.0056$ and $\mathrm{P}=0.0486$, respectively) and showed moderate interfocal variation in paired tumor foci. Methylated promoter status of the two genes was associated with larger tumor size $(\mathrm{P}=0.0110$ and $\mathrm{P}=0.0156$, respectively). Furthermore, aberrant MT1E methylation was more frequent in tumors having necrotic zones $(\mathrm{P}=0.0449)$ or characterized with higher differentiation grade $(\mathrm{P}=0.0144)$, while $M T 1 M$ was more commonly methylated in tumors with higher Fuhrman grade $(\mathrm{P}=0.0272)$. Only unmethylated $M T 1 F$ promoter status was observed in all analyzed samples. Gene expression analysis (51 RCC and 9 NRT) revealed $M T 1 G$ downregulation in tumors $(\mathrm{P}<0.0001)$, while lower MT1E expression levels were associated with the promoter methylation $(\mathrm{P}=0.0077)$. In clear cell RCC, MTIE, MTIG and MTIM expression was higher than that noted in other histological tumor subtypes (all $\mathrm{P}<0.0500$ ). In addition, some associations were observed between metabolic syndrome-related clinical parameters and
\end{abstract}

Correspondence to: Dr Kristina Daniunaite, National Cancer Institute, 1 Santariskiu Street, LT-08406 Vilnius, Lithuania E-mail: kristina.daniunaite@gf.vu.lt

Key words: DNA methylation, diagnostics, metallothionein, MT1E, $M T 1 G, M T 1 M$, renal cell carcinoma promoter methylation or gene expression. In conclusion, the present study revealed the potential role of MTIE and MTIM promoter methylation in RCC development.

\section{Introduction}

Metallothioneins are a family of low molecular weight (6-7 kDa), highly conserved, cysteine-rich non-enzymatic cytosolic proteins which play a vital role in metal ion homeostasis. In humans, the majority of metallothionein genes (16 of 19) are located in a cluster on chromosome 16q13, of which 12 are protein-coding (e.g. MT1A, MT1E and MT1F) and the rest are pseudogenes (e.g. MTICP, MT1L and MT1P1). The main biological roles of metallothioneins in cells are directly related to their ability to bind metal ions. Changes in metallothionein expression alter their major cellular functions, i.e. buffering and delivering zinc and copper, which are critical for proliferating, differentiating and apoptotic cells (1). Due to their affinity for cadmium, lead, or mercury, metallothioneins protect cells from heavy metal toxicity $(2,3)$. They also function as antioxidants against DNA damage caused by free radicals (4).

Metallothioneins have a significant role in cancer development. Gene expression changes have been associated with tumor growth, metastasis and angiogenesis (5). Earlier research has indicated that metallothioneins contribute to the development of resistance to drugs or radiotherapy (6-8); however, recent research has shown that they can also suppress cardiotoxicity induced by anticancer agents (9). Various studies also revealed that metallothionein expression is not universal for different cancer types. Specifically, metallothionein genes are frequently downregulated in liver cancer, squamous cell lung carcinoma, or prostate tumors, which could be associated with promoter DNA methylation (10-12). In contrast, increased metallothionein expression has been reported in melanoma, ovarian or breast tumors (13-15), while investigations of some other tumors produced contradictory results.

Renal cell carcinoma (RCC) comprises $\sim 2-3 \%$ of all non-cutaneous cancers and is the most fatal type of urologic malignancies with high mortality rates in Europe. Lithuania has the third highest RCC incidence rate and is in the first place according to mortality worldwide (16). A variety of histologically distinct tumors falls under the RCC definition, 
with clear cell RCC (ccRCC) being the most common subtype. Other commonly detected tumors are papillary (pRCC) and chromophobe RCC (chRCC). As clinical outcomes are closely related to tumor stage, grade and other parameters, diagnostic methods for accurate cancer characterization, as well as early detection, are critically important. At present, $\sim 50 \%$ of sporadic RCC cases are incidentally detected in asymptomatic patients during examination for other diseases (17). Despite the use of highly sensitive methods, such as computed tomography scan and magnetic resonance imaging, RCC is often detected at already advanced stages when treatment options become limited (18). The lack of diagnostic clinical tests draws attention to genetic and epigenetic features as potential biomarkers of RCC. Novel molecular biomarkers could potentially improve early RCC diagnostics and advise the most beneficial treatment.

In the present study, we investigated aberrant DNA methylation of several protein-coding metallothionein genes aiming to elucidate their importance in renal carcinogenesis. DNA methylation status was compared with clinical-pathological patient characteristics, as well as with gene expression levels. The Cancer Genome Atlas (TCGA) dataset of kidney renal cell carcinoma (KIRC) was used for the screening step. This study led to the identification of the potential importance of $M T I E$ and MTIM genes in RCC development.

\section{Materials and methods}

Patients and samples. In total, 54 patients $[22$ males and 32 females with the mean age of 63 (41-85) and 67 (27-85), respectively] diagnosed with $\mathrm{RCC}$, who underwent full or partial nephrectomy at the National Cancer Institute (Vilnius, Lithuania) between July 2013 and January 2016, were involved in the study. The study cohort mainly consisted of ccRCC and various cases of other histological subtypes (Table I). Tissues were sampled and grades were assigned to tumors by an expert pathologist. The Fuhrman grade describes adverse morphological characteristics of cell nuclei, whereas the differentiation grade, defined according to the WHO recommendations, is based on tissue histology in general. In total, 54 tumors, 10 paired pericancerous renal tissues (PRT; at a distance of $1-2 \mathrm{~cm}$ from the tumor margin), and 33 paired non-cancerous renal tissues (NRT; morphologically normal tissue at $>2 \mathrm{~cm}$ from the tumor margin and $\leq 1 \mathrm{~cm}$ from the surgical margin if partial nephrectomy was performed) were included in the study (Table I). For 10 patients, two tumor foci were available for the molecular analysis. Approval to conduct biomedical research (no. 158200-13-620-192) was obtained from the Lithuanian Bioethics Committee (Vilnius, Lithuania) before initiating the study, and all patients gave informed consent for participation.

Nucleic acid extraction. Renal tissue samples ( $60 \mathrm{mg})$ were mechanically homogenized with cryoPREP ${ }^{\mathrm{TM}} \mathrm{CP} 02$ Impactor using tissueTUBEs TT1 (Covaris, Inc., Woburn, MA, USA). For the isolation of genomic DNA, up to $30 \mathrm{mg}$ of tissue powder was treated with proteinase $\mathrm{K}$ (Thermo Scientific ${ }^{\mathrm{TM}}$; Thermo Fisher Scientific, Vilnius, Lithuania) in $0.5 \mathrm{ml}$ of lysis buffer (50 mM Tris-HCl pH 8.5, 1 mM EDTA, 0.5\% Tween-20; all from Carl Roth $\mathrm{GmbH}$, Co., KG, Karlsruhe, Germany) for up to $18 \mathrm{~h}$ at $55^{\circ} \mathrm{C}$ and DNA was extracted according to the standard phenol-chloroform purification and ethanol precipitation.

The total RNA was extracted using mirVana ${ }^{\mathrm{TM}}$ miRNA Isolation kit (Ambion; Thermo Fisher Scientific, Inc., Waltham, MA, USA) as previously described (12). Briefly, 10 mg of homogenized tissue were treated with $0.5 \mathrm{ml}$ of lysis/binding buffer and $50 \mu \mathrm{l}$ of miRNA homogenate additive for $10 \mathrm{~min}$ in an ice-water bath. The total RNA was extracted with $0.5 \mathrm{ml}$ of acid-phenol:chloroform and purified using the supplied filter cartridges.

Concentration and purity of extracted nucleic acids were evaluated spectrophotometrically with NanoDrop 2000 (NanoDrop Technologies; Thermo Fisher Scientific, Inc.). The integrity of randomly selected DNA samples and all RNA samples was analyzed in 1-1.5\% agarose gels prepared with 1X TAE buffer (Thermo Fisher Scientific, Inc.) and only intact samples were used for the molecular analysis.

DNA methylation analysis. Bisulfite treatment was applied to $400 \mathrm{ng}$ of extracted DNA using EZ DNA Methylation kit (Zymo Research Corp., Irvine, CA, USA) according to the manufacturer's protocol, except that the initial incubation of samples was performed at $42^{\circ} \mathrm{C}$ for $15 \mathrm{~min}$. Methylation-specific PCR (MSP) was used for the qualitative promoter methylation analysis of genes MTIE, MTIG, MTIF and MTIM. Primers specific for methylated or unmethylated DNA (Table II) were designed with Methyl Primer Express Software v1.0 (Applied Biosystems; Thermo Fisher Scientific, Inc.) or selected from the previous publication (12). One microliter of bisulfite-modified DNA was added to $24 \mu \mathrm{l}$ of MSP mix containing 1.25 U AmpliTaq Gold DNA Polymerase, 1X Gold PCR buffer, $1 \mu 1$ of $360 \mathrm{GC}$ Enhancer, $2.5 \mathrm{mM} \mathrm{MgCl}{ }_{2}$ (all from Applied Biosystems; Thermo Fisher Scientific, Inc.), $0.4 \mathrm{mM}$ of each dNTP (Thermo Fisher Scientific, Inc.) and $0.5 \mu \mathrm{M}$ of each primer (Metabion, Martinsried, Germany). Thermocycling conditions were optimized prior to the study and included 35-38 cycles with the primer annealing step at 56-58 ${ }^{\circ} \mathrm{C}$ (Table II). Methylation-positive, methylation-negative, and non-template controls were routinely included. Amplification products were analyzed in 3\% agarose gels with 1X TAE buffer and visualized under UV light after ethidium bromide staining (Carl Roth GmbH, Co., KG).

Gene expression analysis. For cDNA synthesis, $250 \mathrm{ng}$ of extracted RNA was reverse transcribed (RT) using High-Capacity cDNA Reverse Transcription kit with RNase Inhibitor following the manufacturer's protocol (Applied Biosystems; Thermo Fisher Scientific, Inc.). Expression levels of genes MTIE, MTIG, MTIM and endogenous control HPRTI were evaluated by means of quantitative PCR (RT-qPCR) using TaqMan Gene Expression assays (Hs01582977_gH, Hs01584215_g1, Hs00828387_g1, and Hs02800695_m1, respectively; Applied Biosystems; Thermo Fisher Scientific, Inc.). The reaction mix $(20 \mu \mathrm{l})$ consisted of $1 \mathrm{X}$ TaqMan Universal Master Mix II no UNG (Applied Biosystems; Thermo Fisher Scientific, Inc.), 0.4X TaqMan Gene Expression assay and $2 \mu 1$ of RT product. Amplification was performed with Mx3005 $\mathrm{P}^{\mathrm{TM}}$ qPCR System (Agilent Technologies, Inc., Santa Clara, CA, USA) in triplicates per gene. Thermocycling consisted of $95^{\circ} \mathrm{C}$ for $10 \mathrm{~min}$, followed by 45 cycles of $95^{\circ} \mathrm{C}$ 
Table I. Clinicopathological characteristics of the RCC study cohorts.

\begin{tabular}{|c|c|c|c|}
\hline Parameter & $\begin{array}{l}\text { All cases } \\
(n=54)\end{array}$ & $\begin{array}{c}\text { Methylation } \\
\text { analysis }(n=30)\end{array}$ & $\begin{array}{l}\text { Gene expression } \\
\text { analysis }(n=51)\end{array}$ \\
\hline
\end{tabular}

\section{Tissue samples, $\mathrm{n}$}

RCC

PRT

NRT

Histological tumor subtype, $\mathrm{n}$

ccRCC
chRCC
pRCC
OCT
Other types
Tumor size, mean (range), mm
Pathological tumor stage, $\mathrm{n}$
$\leq \mathrm{pT} 2$
$\geq \mathrm{pT} 3$

Fuhrmann grade, $\mathrm{n}$

F2
F3
Unknown
Differentiation grade ${ }^{\mathrm{a}}, \mathrm{n}$
$\leq \mathrm{G} 2$
$\mathrm{G} 3$
Unknown

Necrotic zones in tumor, $\mathrm{n}$

Yes

Sex

Age at diagnosis, mean (range), years

Waist circumference ${ }^{b}$, mean (range), cm

Unknown, $\mathrm{n}$

Raised fasting plasma glucose level ${ }^{\mathrm{c}}, \mathrm{n}$

Yes

No

Female, $\mathrm{n}=32$

67 (27-85)

$102(79-135)$

2

2
1

2

2

Female, $\mathrm{n}=16$

Male, $\mathrm{n}=14$

Female, $\mathrm{n}=30$

67 (27-85)

101 (79-135)

51

0

9

41

3

1

3

3

56 (20-130)

29

22

19

23

9

28

18

5

14

37

Male, $\mathrm{n}=21$

63 (41-85)

100 (78-126)

2

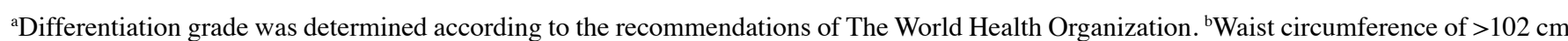
for males and $>88 \mathrm{~cm}$ for females was considered as high. ${ }^{\mathrm{c}}$ Fasting glucose level of $\geq 6.1 \mathrm{mM}$ was considered as high. RCC, renal cell carcinoma; PRT, pericancerous renal tissue; NRT, non-cancerous renal tissue; ccRCC, clear cell RCC; chRCC, chromophobe RCC; pRCC, papillary RCC; OCT, oncocytoma.

for $15 \mathrm{sec}$ and $60^{\circ} \mathrm{C}$ for $1 \mathrm{~min}$. Non-template controls were included in each run. Samples with HPRT1 amplification at cycle $\geq 35$ were considered of low quality and were excluded from the analysis. Data preprocessing was performed using GenEx 6.0.1 software (Multid Analyses AB, Göteborg, Sweden). Relative gene expression values, transformed to a linear scale, were used for statistical analysis.
The Cancer Genome Atlas dataset of renal clear cell carcinoma. For the overview analysis of the metallothionein gene family, the TCGA KIRC dataset was used (19). Global DNA methylation profiling data using Illumina Infinium HumanMethylation450K (HM450) platform and RNA expression data obtained by RNA-seq were utilized in the study. Gene-specific Level 3 datasets were acquired from 
Table II. Primers used for methylation-specific PCR (MSP) and amplification conditions.

\begin{tabular}{|c|c|c|c|c|c|c|c|}
\hline $\begin{array}{l}\text { Gene } \\
\text { symbol }\end{array}$ & $\begin{array}{c}\text { Primer } \\
\text { ID }\end{array}$ & Primer sequence $\left(5^{\prime}-3^{\prime}\right)$ & $\begin{array}{l}\text { Product } \\
\text { size (bp) }\end{array}$ & $\begin{array}{l}\text { Amplicon } \\
\text { location from } \\
\text { TSS (bp) }\end{array}$ & $\begin{array}{c}\text { PCR } \\
\text { cycles }\end{array}$ & $\begin{array}{c}\text { Primer } \\
\text { annealing } \\
\mathrm{T},\left({ }^{\circ} \mathrm{C}\right)\end{array}$ & (Refs.) \\
\hline \multirow[t]{4}{*}{$M T 1 E$} & $\mathrm{M}-\mathrm{F}$ & GGATTTCGGGAATATCGC & 217 & $-113 /+104$ & 38 & 56 & (12) \\
\hline & M-R & ACGAAAATCGAACCGAAC & & & & & \\
\hline & U-F & TTTGGATTTTGGGAATATTGT & 220 & $-116 /+104$ & & & \\
\hline & U-R & ACAAAААТСАААССАААСАСА & & & & & \\
\hline \multirow[t]{4}{*}{$M T 1 F$} & $\mathrm{M}-\mathrm{F}$ & GTATTCGGAATTTTAAGGGGC & 134 & $-262 /-129$ & 35 & 57 & This study \\
\hline & M-R & CGAACCGTCCCTTTAAAATC & & & & & \\
\hline & U-F & TAGGTATTTGGAATTTTAAGGGGT & 139 & $-265 /-127$ & & & \\
\hline & U-R & CACAААССАТСССТТТААААТС & & & & & \\
\hline \multirow[t]{4}{*}{$M T 1 G$} & $\mathrm{M}-\mathrm{F}$ & TCGTATACGGGGGGTATAGC & 131 & $-232 /-102$ & 37 & 58 & This study \\
\hline & M-R & GCGATCCCGACCTAAACT & & & & & \\
\hline & $\mathrm{U}-\mathrm{F}$ & AAGTTGTATATGGGGGGTATAGT & 137 & $-235 /-99$ & & & \\
\hline & U-R & СССАСААТСССААССТАААСТ & & & & & \\
\hline \multirow[t]{4}{*}{$M T 1 M$} & $\mathrm{M}-\mathrm{F}$ & GGATATTGCGTATTATTCGGC & 112 & $-240 /-129$ & 38 & 56 & This study \\
\hline & M-R & ATAAATACCGAACGCACCATC & & & & & \\
\hline & U-F & TTGGGGATATTGTGTATTATTTGGT & 116 & $-244 /-129$ & & & \\
\hline & U-R & ATAAATACCAAACACACCATCCC & & & & & \\
\hline
\end{tabular}

TSS, transcription start site; M/U, primer specific for methylated/unmethylated sequence; $\mathrm{F} / \mathrm{R}$, forward/reverse primer.

the cBioPortal (http://www.cbiopotal.org) and MethHC (http://methhc.mbc.nctu.edu.tw) data analyses portals in September $2018(20,21)$.

Statistical analysis. Statistical analysis was performed using STATISTICA v8.0 (StatSoft Inc., Tulsa, OK, USA). The two-sided Fisher's exact test was used for two-group comparisons of categorical data, while the Mann-Whitney U test was used for continuous data. Heterogeneity index (HI) was calculated to estimate the discordance rate of methylation status of paired tumor foci. Correlations of gene expression levels with quantitative clinicopathological or molecular parameters were evaluated by calculating Spearman's $R_{S}$ and/or Pearson's $R_{P}$ correlation coefficients. Receiver Operating Characteristic (ROC) curve analysis was performed and the area under the curve (AUC) was calculated in order to evaluate the clinical utility of the test. Logistic regression analysis was applied for the putative biomarker combination. P-level of $<0.0500$ was considered significant. Data visualization was developed using GraphPad Prism v5.03 software (GraphPad Software, Inc., La Jolla, CA, USA).

\section{Results}

Metallothionein gene analysis in the TCGA dataset. For the screening step, DNA methylation and gene expression data were extracted for 8 protein-coding metallothionein genes from the TCGA KIRC dataset. In total, data of $520 \mathrm{ccRCC}$ and 209 healthy tissue samples were available for the analysis. Due to the ubiquitous expression of some of the metallothioneins in various tissues and induction by a variety of stimuli (including hormones and growth factors), we focused on $M T 1$ group genes only. Significant differences in methylation levels between ccRCC and healthy tissues were identified for 5 genes, of which MT1A, MT1E, MTIF and MT1M had higher methylation levels in tumors, while $M T 1 B$ was highly, but still differentially methylated in both tissue types (all $\mathrm{P}<0.0500$; Fig. 1). Furthermore, despite the variable range, the methylation intensity of MT1A, MTIE, MTIF and MTIM was correlated with downregulated gene expression (all $\mathrm{P}<0.0500$; Fig. 2). Expression of $M T 1 B$ was absent in almost all tumor samples, therefore, it could not be compared with the promoter methylation (data not shown).

DNA methylation analysis of selected metallothionein genes. Based on the TCGA KIRC dataset analysis and with regard to the literature review, four metallothionein genes, $M T 1 E, M T 1 F$, $M T 1 G$, and $M T 1 M$, were selected for the qualitative analysis of promoter DNA methylation. MT1E, MT1G, and MT1M were methylated in $\leq 43.3 \%$ of tumors and less frequently in NRT, but only MTIE and MT1M showed significant differences $(\mathrm{P}=0.0056$ and $\mathrm{P}=0.0486$, respectively). The three genes were also methylated in PRT indicating the field cancerization phenomenon of the tumor-adjacent area (Fig. 3A). Interfocal variation of methylation status was present in $M T 1 E$ and $M T 1 M$ gene promoters, but no heterogeneity of $M T 1 G$ and $M T 1 F$ was detected (Fig. 3B). Only unmethylated promoter status of $M T 1 F$ was observed in all analyzed tissues.

In our cohort, the sensitivity and specificity of the $M T 1 E$ and $M T 1 M$ gene combination were 53.3 and $83.3 \%$, respectively. The ROC curve analysis of the TCGA KIRC dataset revealed comparable diagnostic values of the two genes 


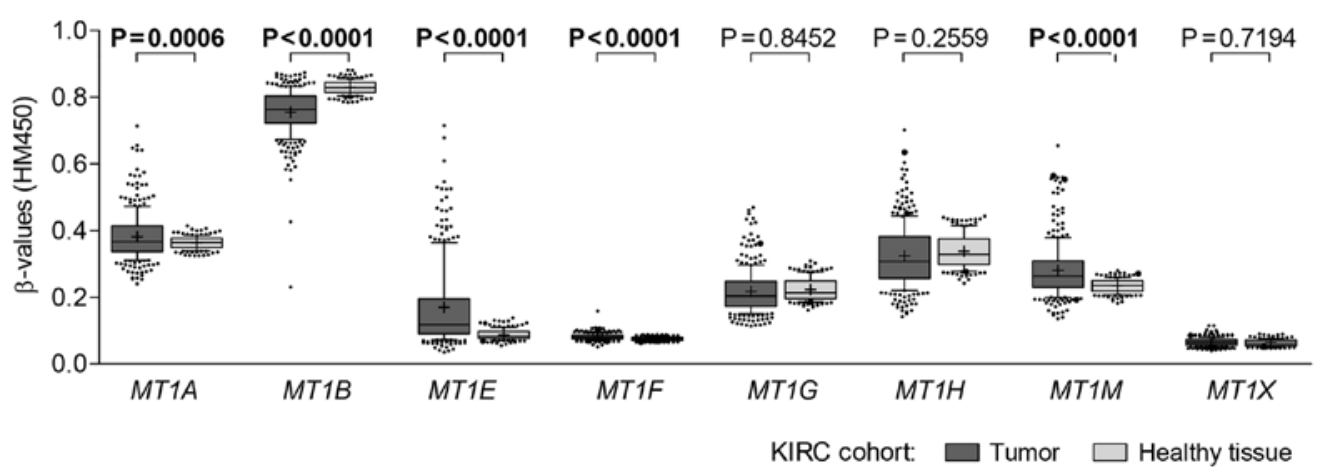

Figure 1. DNA methylation levels of selected protein-coding metallothionein genes in the renal clear cell carcinoma (KIRC) cohort of The Cancer Genome Atlas (TCGA). Level 3 DNA methylation data, obtained using Illumina Human Methylation450K (HM450) platform, was used to generate the plots. The box extends from the 25 to 75 th percentiles; the line in the middle of the box is plotted at median; the plus sign depicts the mean; the whiskers represent the 10-90\% range; data values outside the range are marked as dots. Significant P-values are in bold.
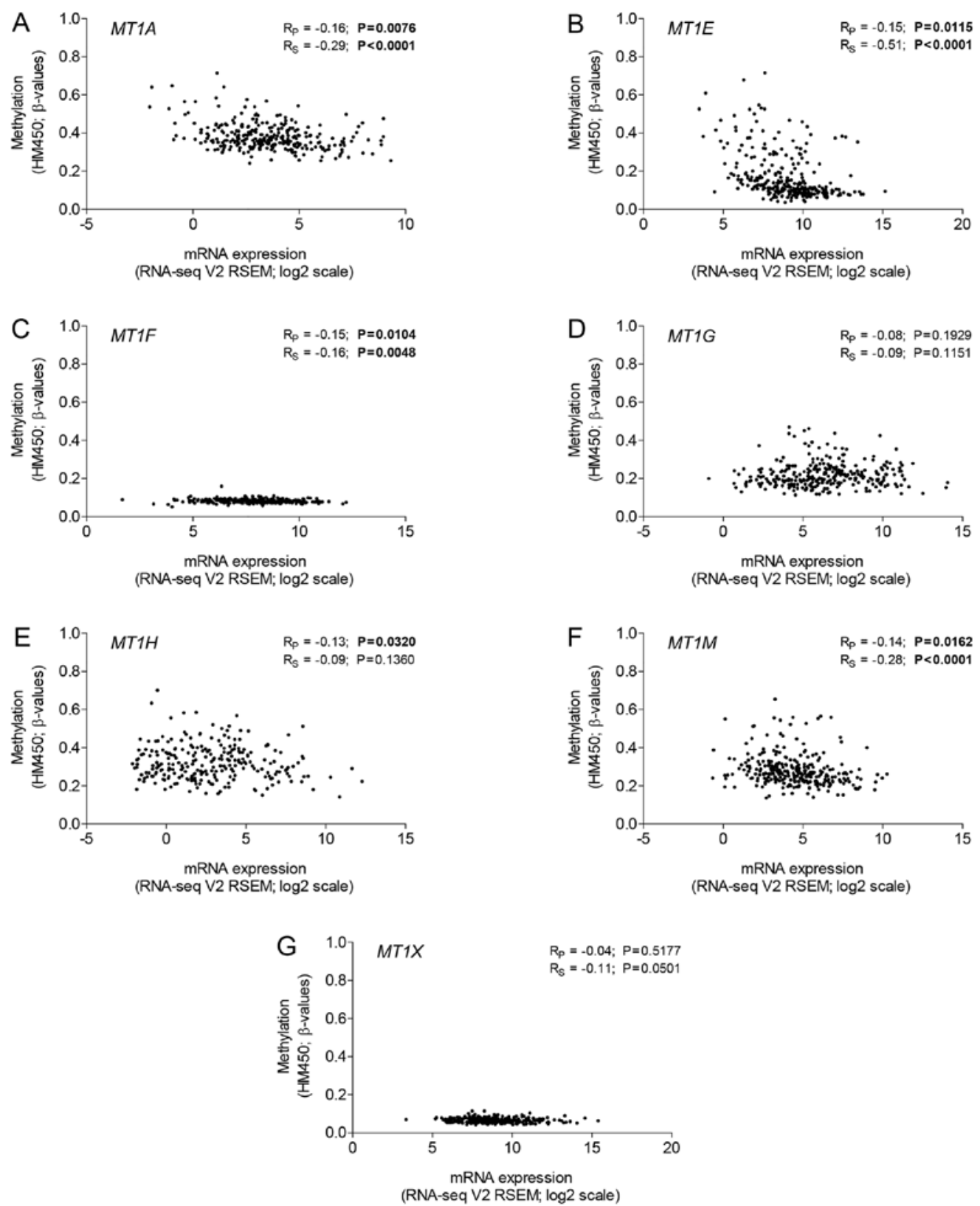

Figure 2. Correlations between promoter methylation and gene expression for (A) $M T 1 A$, (B) $M T 1 E$, (C) $M T 1 F$, (D) $M T 1 G$, (E) $M T 1 H$, (F) $M T 1 M$ and (G) MTIX in the renal clear cell carcinoma (KIRC) cohort of The Cancer Genome Atlas (TCGA). Level 3 DNA methylation data, obtained using Illumina HumanMethylation450K (HM450) platform, and level 3 KIRC RNA-seq RSEM data were used to generate scatter plots. RNA-seq data is plotted on log2 scale. Pearson's R $\left(R_{\mathrm{P}}\right)$ and Spearman's $\mathrm{R}\left(\mathrm{R}_{\mathrm{S}}\right)$ correlation coefficients are provided with respective P-values. Significant P-values are in bold. 

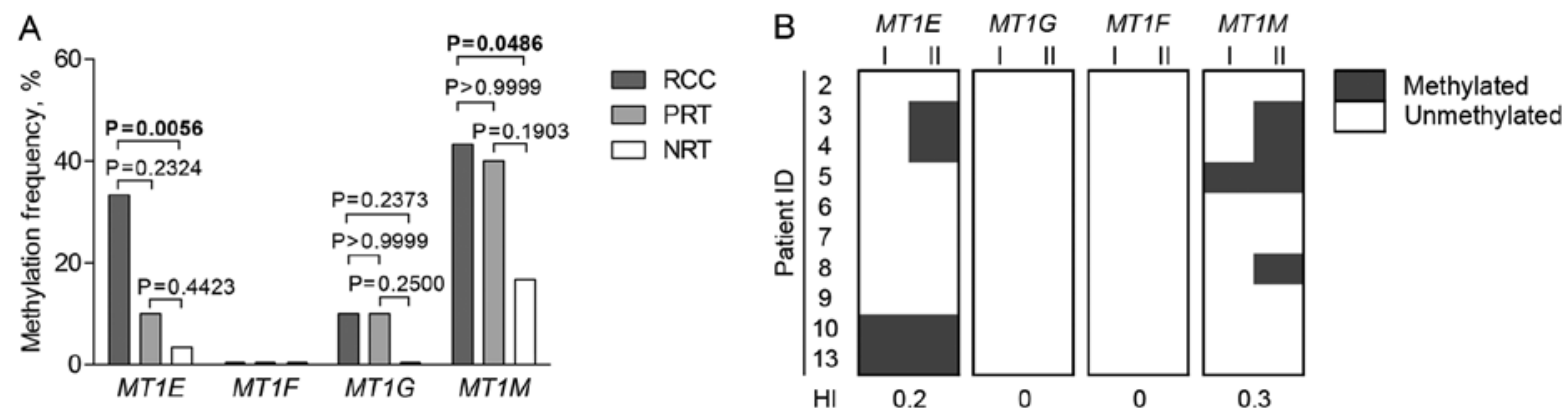

Figure 3. DNA methylation status of genes $M T I E, M T 1 F, M T 1 G$ and $M T 1 M$ in renal cancer. (A) Promoter DNA methylation frequencies in tumors (RCC), pericancerous (PRT) and non-cancerous (NRT) renal tissues. (B) Heterogeneity of methylation status in multifocal (I and II foci) carcinoma cases. HI, heterogeneity index. Significant P-values are in bold.
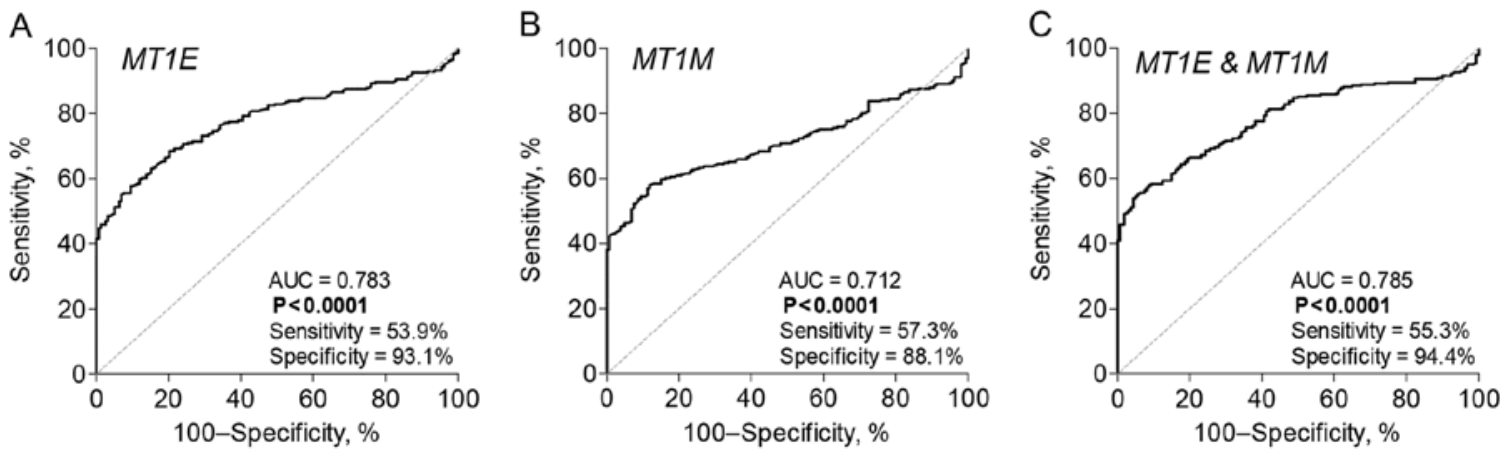

Figure 4. Receiver operating characteristic (ROC) curve analysis of promoter methylation of the selected metallothionein genes in the renal clear cell carcinoma (KIRC) cohort of The Cancer Genome Atlas (TCGA). (A) MTlE gene; (B) MTlM gene; (C) combination of MTlE and MTIM. Sensitivity, specificity and area under the curve (AUC) values are provided. Significant P-values are in bold.
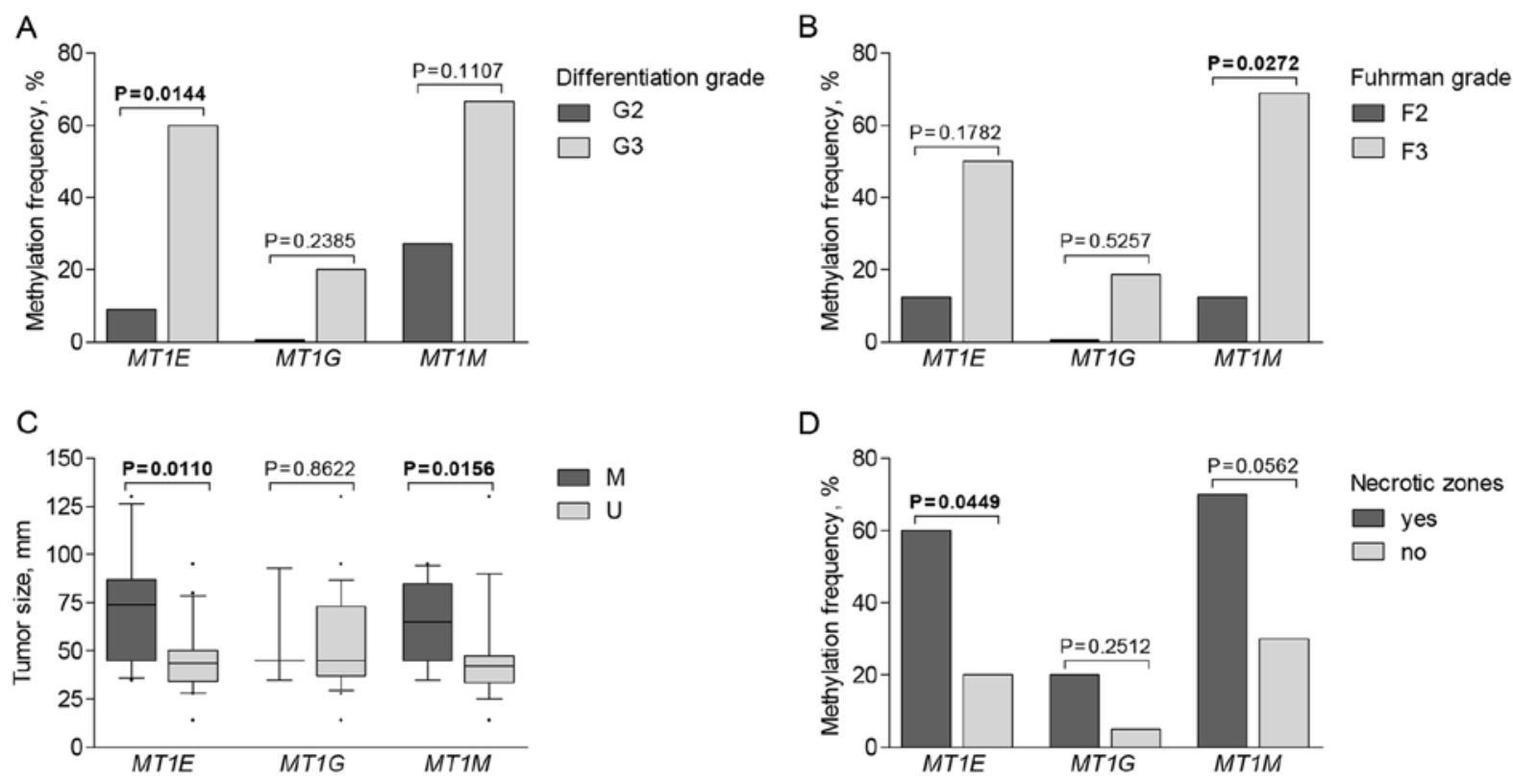

D

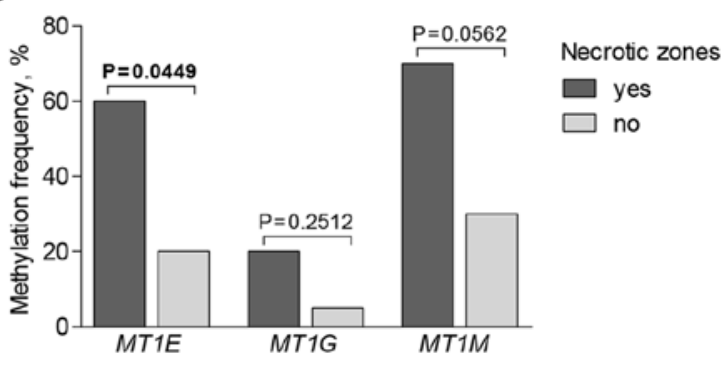

Figure 5. Associations of metallothionein gene promoter methylation in renal tumors (RCC) and clinicopathological patient characteristics. (A) Methylation frequencies according to tumor differentiation grade; (B) methylation frequencies according to Fuhrmann grade; (C) distribution of tumor size according to gene methylation status; (D) methylation frequencies according to the presence of necrotic zones in tumor. The box extends from the 25 to 75 th percentiles; the line in the middle of the box is plotted at median; the whiskers represent the 10-90\% range; data values outside the range are marked as dots. $\mathrm{M} / \mathrm{U}$, methylated/unmethylated gene promoter status. Significant P-values are in bold.

individually or in combination, with the specificity reaching up to $94.4 \%$ (Fig. 4).
DNA methylation and clinicopathological parameters. Aberrant promoter methylation of MTIE, MTIG and MTIM 
A
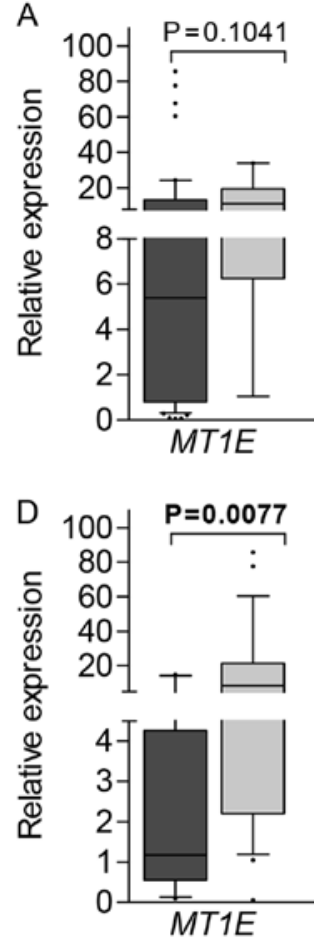

G

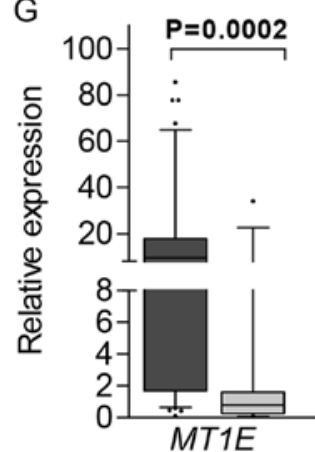

B

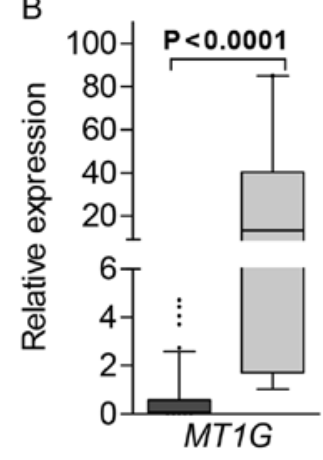

E

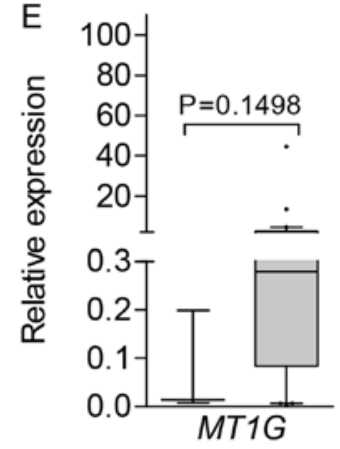

H

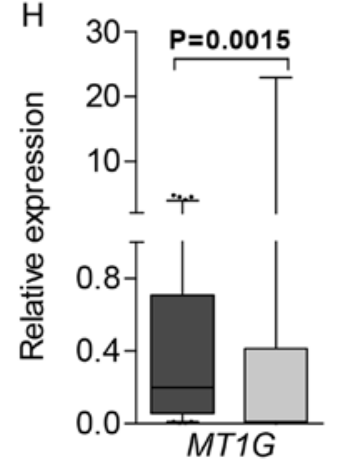

C
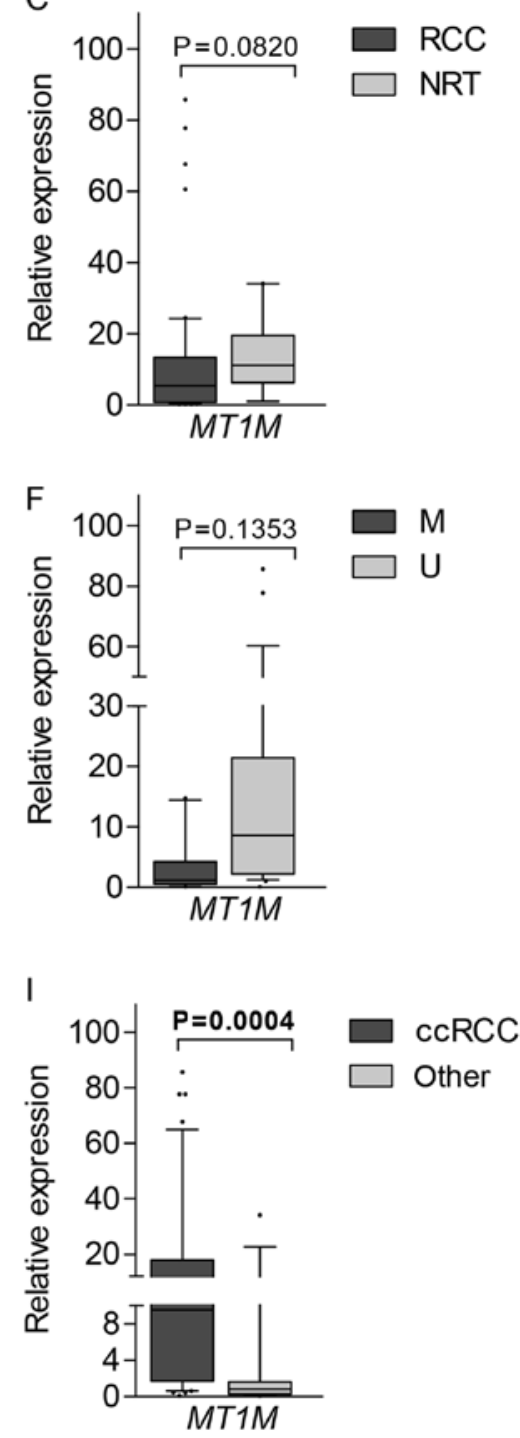

Figure 6. Relative expression of metallothionein genes in renal tissues. (A-C) Gene expression in renal tumors (RCC) and non-cancerous renal tissues (NRT). (D-F) Gene expression in renal tissues according to promoter methylation status. (G-I) Gene expression in RCC according to histological tumor subtypes. The box extends from the 25 to 75 th percentiles; the line in the middle of the box is plotted at median; the whiskers represent the $10-90 \%$ range; data values outside the range are marked as dots. M/U, methylated/unmethylated gene promoter; ccRCC, clear cell RCC. Significant P-values are in bold.

was further analyzed according to clinicopathological patient characteristics. Methylation was more commonly detected in cases with advanced disease parameters. Higher MTIE methylation frequency was observed in tumors with higher differentiation grade $(\mathrm{P}=0.0144)$, while $M T 1 M$ was more commonly methylated in RCC cases characterized with higher Fuhrman grade ( $\mathrm{P}=0.0272$; Fig. 5A and B). Tumors with methylated $M T 1 E$ and $M T 1 M$ promoters were significantly larger than those with unmethylated promoter status $(\mathrm{P}=0.0110$ and $\mathrm{P}=0.0156$, respectively; Fig. 5C). Furthermore, methylation of metallothionein genes was recurrently observed in tumors having necrotic zones; however, only MT1E showed significant difference ( $\mathrm{P}=0.0449$; Fig. 5D). No associations were detected between metallothionein gene methylation and patient age, sex, or pathological tumor stage (data not shown).

Gene expression analysis and association with DNA methylation. Gene expression at the transcriptional level was quantified by means of RT-qPCR. Lower expression levels of MT1E,
$M T 1 G$ and MT1M were detected in tumors as compared to NRT samples, however, only $M T 1 G$ showed significant difference $(\mathrm{P}<0.0001$; Fig. 6A-C). Downregulation of MT1E correlated with methylated promoter status $(\mathrm{P}=0.0077)$, while no such associations were detected for MTIG and MT1M (both $\mathrm{P}>0.0500$; Fig. 6D-F). All three genes were expressed at significantly higher levels in ccRCC in comparison to the mixed group of other histological tumor subtypes $(\mathrm{P}=0.0002$, $\mathrm{P}=0.0015$ and $\mathrm{P}=0.0004$ for MTIE, MTIG and MT1M, respectively; Fig. 6G-I). No other associations were observed between gene expression and clinical-pathological parameters.

Associations with metabolic syndrome-related parameters. Metabolic syndrome is concomitant with particular tumor types and is considered to be a risk factor of kidney cancer. In the present study, molecular alterations of metallothionein genes were associated with particular parameters used to diagnose metabolic syndrome. Methylated MTIE status in ccRCC subtype was more common in cases with raised fasting 
A

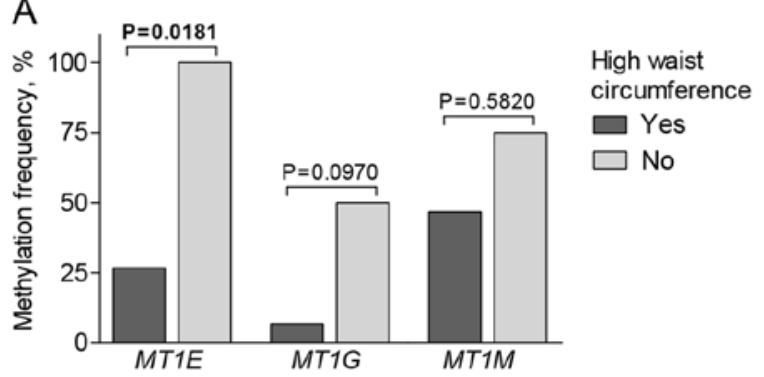

B

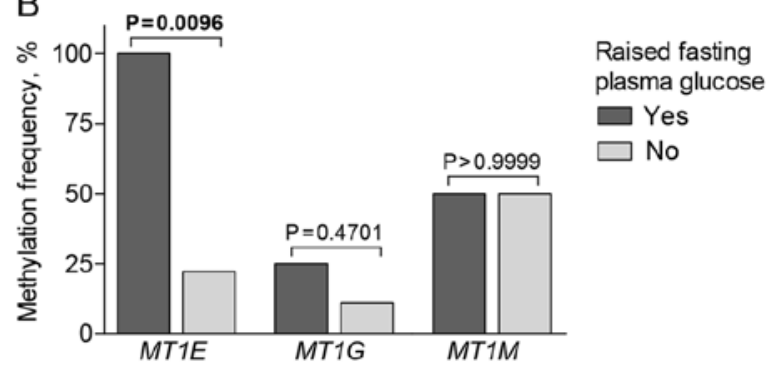

Figure 7. DNA methylation of metallothionein genes in renal clear cell tumors (ccRCC) according to metabolic syndrome-associated characteristics. (A) Methylation frequencies according to waist circumference. (B) Methylation frequencies according to fasting plasma glucose level. Significant P-values are in bold.

plasma glucose level $(\mathrm{P}=0.0096)$, but less frequent in patients with high waist circumference $(\mathrm{P}=0.0181$; Fig. 7). Notably, expression of $M T 1 G$ and $M T 1 M$ was positively correlated with women's waist circumference $\left(\mathrm{R}_{\mathrm{S}}=0.38, \mathrm{P}=0.0490\right.$ and $\mathrm{R}_{\mathrm{S}}=0.47, \mathrm{P}=0.0119$, respectively), whereas $M T 1 M$ was upregulated in men having raised fasting glucose level $(\mathrm{P}=0.0348$; data not shown). Due to the missing data for the majority of the cases, associations with other metabolic syndrome-related parameters (such as hypertriglyceridemia or high-density lipoprotein level) were not analyzed.

\section{Discussion}

In recent years, metallothioneins have emerged as important players in human carcinogenesis. Due to their unique function of metal ion buffering and delivery in a cell, these small cysteine-rich proteins have been shown to be pivotal regulators of various cellular processes, such as proliferation, differentiation, or apoptosis. Besides metal ion homeostasis and detoxification, metallothioneins protect cells against oxidative stress and DNA damage by scavenging free radicals. Numerous studies have reported deregulation of metallothionein expression in human tumors and, thus, their important role in various aspects of carcinogenesis has been proposed (10-15). However, the mechanisms responsible for the deregulated expression have been sparsely investigated.

In the present study, we investigated several protein-coding metallothionein genes in renal tumors aiming to evaluate their promoter DNA methylation for potential clinical utility. Four metallothionein genes, namely MT1E, MT1G, MTIF and $M T 1 M$, were selected for epigenetic analysis in renal cell carcinoma (RCC) and paired non-cancerous renal tissues. To the best of our knowledge, this is the first study to report aberrant methylation of $M T 1 E$ and $M T 1 M$ genes in RCC. We showed that methylation of MT1E and MT1M genes was tumor-specific, which indicates the potential clinical value of the two biomarkers in RCC diagnostics. Moreover, these results were supported by our preliminary observations made by analyzing the TCGA KIRC cohort (19). Until now, the two genes have been investigated in several other cancer types mostly at transcriptional and/or translational levels; however, the data concerning their promoter methylation are limited. In the present study, MTIE methylation associated with downregulated gene expression was observed in RCC, which is in accordance with our previous results obtained in prostate tumors (12). Other studies have also reported epigenetic silencing of MT1E in endometrial tumors, melanoma, and several other cancer localizations $(22,23)$. The presence of $M T 1 E$ methylation in metastases of melanoma patients, as well as in several invasive melanoma cell lines, suggest its potential involvement in cancer progression (23). In this study, MTIE was more frequently methylated in tumors of higher differentiation grade, larger size, or having necrotic zones, all of which are indicative of advanced disease. Furthermore, MT1E methylation status was associated with patient waist circumference and raised fasting plasma glucose, i.e. two of the parameters used for diagnosing metabolic syndrome, which is considered as one of the RCC risk factors. This hints that MTIE methylation may be an early event in renal carcinogenesis and, thus, lays the grounds for future investigations.

$M T 1 M$ was the most commonly methylated gene in our cohort (43\%). Its aberrant methylation was also observed in pericancerous renal tissues (PRT) $(40 \%)$ indicating the field cancerization phenomena in RCC, i.e. when histologically normal tissue adjacent to cancer is primed to undergo transformation (24). Promoter methylation of MTIE and MTIG was also present in tumor-surrounding tissues suggesting that such epigenetic alterations might precede the development of RCC and predispose to multifocal tumors. In clinical practice, detection of aberrant methylation in normal-appearing biopsy specimens may be indicative of a missed cancerous lesion nearby and could justify the need for a repeat biopsy. Furthermore, together with $M T 1 E$, interfocal heterogeneity of MT1M methylation status was observed in several RCC cases, which is most likely attributable to discrepant grades of different tumor foci. To date, epigenetic analysis of MT1M in RCC has not been reported; however, it is one of the most studied metallothionein genes in various other cancer types. Downregulation of MT1M has been observed in hepatocellular, esophagus squamous cell carcinomas, breast, and other tumors and was associated with various clinicopathological parameters describing cancer aggressiveness (25-27). In this study, MT1M methylation was more frequently detected in tumors of larger size and higher Fuhrman grade, which is in accordance with previous observations in other tumors reporting its putative role in cancer progression $(25,27,28)$.

In the present study, methylation of $M T 1 G$ and $M T 1 F$ was rare or absent. However, $M T 1 G$ was the only metallothionein with significantly decreased gene expression in RCC as compared to healthy tissues. Aberrant $M T 1 G$ methylation as a 
novel biomarker of RCC was first reported by Dalgin et al (29). In previous studies, $M T 1 G$ downregulation and epigenetic silencing have been associated with poor clinical outcome and/or drug resistance in various tumors $(6,30,31)$. In addition, another mechanism, loss of heterozygosity, has been reported as being potentially responsible for the downregulated expression (32); however, it was not evaluated in the present study. We did not detect any correlations between $M T 1 G$ promoter methylation or transcriptional expression and clinicopathological variables, which may be related to the low $M T 1 G$ methylation frequency observed in our cohort. As no aberrant methylation of MTIF was detected in any renal tissues, this gene was omitted from our gene expression analysis. According to recent studies (5), MT1F overexpression rather than downregulation seems to be more commonly observed in cancer, which is in agreement with the lack of promoter methylation in our data.

Previous studies have demonstrated that metallothionein expression is quite specific to tumor localization as summarized by Si et al (5). Even in the same type of cancer, results can be contradictory due to the unique tumor microenvironment and varying external stimuli. In this study, despite promoter methylation status, $M T 1 E, M T 1 G$, and $M T 1 M$ were expressed at significantly higher levels in ccRCC as compared to other histological subtypes of renal tumors. Considering their previously reported role in drug resistance, expression and/or methylation analysis of the three metallothionein genes in renal tumors could potentially provide additional information for treatment decision making.

In conclusion, this study has revealed the potential clinical value of aberrant promoter methylation of metallothionein genes in RCC. Methylated promoter status of MT1E and $M T 1 M$, together with other clinicopathological disease parameters, may serve for more accurate RCC characterization and personalized treatment selection at the time of diagnosis. However, further validation of these putative biomarkers are needed in larger independent cohorts, including liquid-biopsy samples, such as plasma or urine. DNA methylation analysis in body fluids not only enables patient monitoring by acquiring serial samples but is also considered to better reflect all tumor foci (including metastases), unlike tissue biopsy, which poorly accounts for RCC heterogeneity. As the diversity of metallothionein functions in the cell has been coming to light of late, functional analysis may provide significant insights on how the observed epigenetic deregulation is translated to the protein level, and also may indicate potential targets for drug development.

\section{Acknowledgements}

The authors would like to thank the patients who participated in this study and the staff of National Center of Pathology for tissue sampling and histopathologic evaluation. We also thank Mr. Kerry S. Keys for language editing. The Cancer Genome Atlas project (http://cancergenome.nih.gov) is acknowledged for data availability.

\section{Funding}

The present study was supported by the grant no. S-MIP-17-54 from the Research Council of Lithuania. RM and KD were supported by the European Social Fund according to the activity 'Development of students' ability to carry out R\&D activities' under Measure No. 09.03.3-LMT-K-712 'Development of Scientific Competences of Scientists, other Researchers and Students through Practical Research Activities' (grant no. 09.03.3-LMT-K-712-09-0156 to KD).

\section{Availability of data and materials}

The RCC datasets analyzed in the current study are available from the corresponding author on reasonable request. The KIRC dataset used for general epigenetic overview of the metallothionein gene family is publicly available from the TCGA Research Network (http://cancergenome.nih.gov/).

\section{Authors' contributions}

RM performed the experiments and the data analysis, and drafted the manuscript. AZ and AB collected and analyzed the clinical data. FJ coordinated the patient selection, supervised the clinical data analysis and was involved in the conception of the study. SJ contributed to study implementation, supplied the samples, and participated in establishing research schemes. KD conceived and designed the study, supervised the experimental part of the study, and was a major contributor in writing the manuscript. All authors read and approved the manuscript and agree to be accountable for all aspects of the research in ensuring that the accuracy or integrity of any part of the work are appropriately investigated and resolved.

\section{Ethics approval and consent to participate}

Approval to conduct biomedical research (no. 15820013-620-192) was obtained from the Lithuanian Bioethics Committee (Vilnius, Lithuania) before initiating the study, and all patients provided informed consent for participation.

\section{Patient consent for publication}

Not applicable.

\section{Competing interests}

The authors declare that they have no competing interests.

\section{References}

1. Krężel A and Maret W: The functions of metamorphic metallothioneins in zinc and copper metabolism. Int J Mol Sci 18: E1237, 2017.

2. Klaassen CD, Liu J and Diwan BA: Metallothionein protection of cadmium toxicity. Toxicol Appl Pharmacol 238: 215-220, 2009.

3. Agrawal S, Flora G, Bhatnagar P and Flora SJ: Comparative oxidative stress, metallothionein induction and organ toxicity following chronic exposure to arsenic, lead and mercury in rats. Cell Mol Biol 60: 13-21, 2014.

4. Dutsch-Wicherek M, Sikora J and Tomaszewska R: The possible biological role of metallothionein in apoptosis. Front Biosci 13: 4029-4038, 2008.

5. Si M and Lang J: The roles of metallothioneins in carcinogenesis: J Hematol Oncol 11: 107, 2018.

6. Sun X, Niu X, Chen R, He W, Chen D, Kang R and Tang D: Metallothionein-1G facilitates sorafenib resistance through inhibition of ferroptosis. Hepatology 64: 488-500, 2016. 
7. Gansukh T, Donizy P, Halon A, Lage H and Surowiak P: In vitro analysis of the relationships between metallothionein expression and cisplatin sensitivity of non-small cellular lung cancer cells. Anticancer Res 33: 5255-5260, 2013.

8. Smith DJ, Jaggi M, Zhang W, Galich A, Du C, Sterrett SP, Smith LM and Balaji KC: Metallothioneins and resistance to cisplatin and radiation in prostate cancer. Urology 67: 1341-1347, 2006.

9. Jing L, Yang M, Li Y, Yu Y, Liang B, Cao L, Zhou X, Peng S and Sun Z: Metallothionein prevents doxorubicin cardiac toxicity by indirectly regulating the uncoupling proteins 2 . Food Chem Toxicol 110: 204-213, 2017.

10. Park Y and Yu E: Expression of metallothionein-1 and metallothionein-2 as a prognostic marker in hepatocellular carcinoma. J Gastroenterol Hepatol 28: 1565-1572, 2013.

11. Theocharis S, Karkantaris C, Philipides T, Agapitos E, Gika A Margeli A, Kittas C and Koutselinis A: Expression of metallothionein in lung carcinoma: Correlation with histological type and grade. Histopathology 40: 143-151, 2002.

12. Demidenko R, Daniunaite K, Bakavicius A, Sabaliauskaite R, Skeberdyte A, Petroska D, Laurinavicius A, Jankevicius F, Lazutka JR and Jarmalaite S: Decreased expression of MTIE is a potential biomarker of prostate cancer progression. Oncotarget 8: 61709-61718, 2017.

13. Weinlich G, Eisendle K, Hassler E, Baltaci M, Fritsch PO and Zelger B: Metallothionein-overexpression as a highly significant prognostic factor in melanoma: A prospective study on 1,270 patients. Br J Cancer 94: 835-841, 2006.

14. Hengstler JG, Pilch H, Schmidt M, Dahlenburg H, Sagemüller J, Schiffer I, Oesch F, Knapstein PG, Kaina B and Tanner B: Metallothionein expression in ovarian cancer in relation to histopathological parameters and molecular markers of prognosis. Int J Cancer 95: 121-127, 2001.

15. Tai SK, Tan OJ, Chow VT, Jin R, Jones JL, Tan PH, Jayasurya A and Bay BH: Differential expression of metallothionein 1 and 2 isoforms in breast cancer lines with different invasive potential: Identification of a novel nonsilent metallothionein-1H mutant variant. Am J Pathol 163: 2009-2019, 2003.

16. Wong MCS, Goggins WB, Yip BHK, Fung FDH, Leung C, Fang Y, Wong SYS and Ng CF: Incidence and mortality of kidney cancer: Temporal patterns and global trends in 39 countries. Sci Rep 7: 15698, 2017.

17. Rabjerg M, Mikkelsen MN, Walter S and Marcussen N: Incidental renal neoplasms: Is there a need for routine screening? A Danish single-center epidemiological study. APMIS 122: 708-714, 2014.

18. Linehan WM, Bratslavsky G, Pinto PA, Schmidt LS, Neckers L, Bottaro DP and Srinivasan R: Molecular diagnosis and therapy of kidney cancer. Annu Rev Med 61: 329-343, 2010.

19. Ricketts CJ, De Cubas AA, Fan H, Smith CC, Lang M, Reznik E, Bowlby R, Gibb EA, Akbani R, Beroukhim R, et al: The cancer genome atlas comprehensive molecular characterization of renal cell carcinoma. Cell Rep 23: 313-326, 2018.
20. Cerami E, Gao J, Dogrusoz U, Gross BE, Sumer SO, Aksoy BA, Jacobsen A, Byrne CJ, Heuer ML, Larsson E, et al: The cBio cancer genomics portal: An open platform for exploring multidimensional cancer genomics data. Cancer Discov 2: 401-404, 2012.

21. Huang WY, Hsu SD, Huang HY, Sun YM, Chou CH, Weng SL and Huang HD: MethHC: A database of DNA methylation and gene expression in human cancer. Nucleic Acids Res 43: D856-D861, 2015.

22. Tse KY, Liu VW, Chan DW, Chiu PM, Tam KF, Chan KK, Liao XY, Cheung AN and Ngan HY: Epigenetic alteration of the metallothionein 1E gene in human endometrial carcinomas. Tumour Biol 30: 93-99, 2009.

23. Faller WJ, Rafferty M, Hegarty S, Gremel G, Ryan D, Fraga MF, Esteller M, Dervan PA and Gallagher WM: Metallothionein 1E is methylated in malignant melanoma and increases sensitivity to cisplatin-induced apoptosis. Melanoma Res 20: 392-400, 2010.

24. Chai $\mathrm{H}$ and Brown RE: Field effect in cancer-an update. Ann Clin Lab Sci 39: 331-337, 2009.

25. Ding J and Lu SC: Low metallothionein $1 \mathrm{M}$ expression association with poor hepatocellular carcinoma prognosis after curative resection. Genet Mol Res 15, 2016.

26. Oka D, Yamashita S, Tomioka T, Nakanishi Y, Kato $H$, Kaminishi $\mathrm{M}$ and Ushijima T: The presence of aberrant DNA methylation in noncancerous esophageal mucosae in association with smoking history: A target for risk diagnosis and prevention of esophageal cancers. Cancer 115: 3412-3426, 2009.

27. Jadhav RR, Ye Z, Huang RL, Liu J, Hsu PY, Huang YW, Rangel LB, Lai HC, Roa JC, Kirma NB, et al: Genome-wide DNA methylation analysis reveals estrogen-mediated epigenetic repression of metallothionein-1 gene cluster in breast cancer. Clin Epigenetics 7: 13, 2015.

28. Fu CL, Pan B, Pan JH and Gan MF: Metallothionein $1 \mathrm{M}$ suppresses tumorigenesis in hepatocellular carcinoma. Oncotarget 8: 33037-33046, 2017.

29. Dalgin GS, Drever M, Williams T, King T, DeLisi C and Liou LS: Identification of novel epigenetic markers for clear cell renal cell carcinoma. J Urol 180: 1126-1130, 2008.

30. Guo R, Wu G, Li H, Qian P, Han J, Pan F, Li W, Li J and Ji F: Promoter methylation profiles between human lung adenocarcinoma multidrug resistant A549/cisplatin (A549/DDP) cells and its progenitor A549 cells. Biol Pharm Bull 36: 1310-1316, 2013.

31. Nguyen A, Jing Z, Mahoney PS, Davis R, Sikka SC, Agrawal KC and Abdel-Mageed AB: In vivo gene expression profile analysis of metallothionein in renal cell carcinoma. Cancer Lett 160: 133-140, 2000

32. Chan KY, Lai PB, Squire JA, Beheshti B, Wong NL, Sy SM and Wong N: Positional expression profiling indicates candidate genes in deletion hotspots of hepatocellular carcinoma. Mod Pathol 19: 1546-1554, 2006. 